\title{
SFBC DESIGN TRADEOFFS FOR MOBILE SC-FDMA WITH APPLICATION TO LTE-ADVANCED
}

\author{
Balachander Narasimhan, Naofal Al-Dhahir, Hlaing Minn \\ University of Texas at Dallas \\ Deptartment of Electrical Engineering \\ Richardson, TX-75080
}

\begin{abstract}
Single-carrier frequency-division multiple-access (SC-FDMA) has been adopted in the uplink of the LTE standard due to its lower peak-to-average-power ratio (PAPR) compared to orthogonal frequency-division multiple access (OFDMA). Recent activities in the LTE-advanced (LTE-A) standardization have focused on effective uplink transmit diversity schemes with low PAPR [1] but without considering the performance degradation due to Doppler despite the fact that LTE-A is required to support high mobility. In this paper, we present an open-loop space-frequency block coding (SFBC) scheme for the SC-FDMA uplink and demonstrate its robustness to high Doppler and large multipath delay spread while enjoying full spatial diversity, low PAPR and practical decoding complexity by a suitable design of the frequency span of each SFBC codeword. In this paper, we study the design tradeoffs involved in SFBC design for mobile SC-FDMA.
\end{abstract}

Index Terms - SC-FDMA, Alamouti, SFBC, PAPR, mobility

\section{INTRODUCTION}

SC-FDMA is an attractive multiple access scheme for broadband wireless communications because it enjoys the same advantage of OFDMA of having low equalization complexity, but does not suffer from its major drawback; namely, high PAPR which affects the battery life and performance of a mobile terminal. SC-FDMA has been specified as the mode of uplink transmission in the current LTE standard [2]. Moreover, in the upcoming LTE-Advanced (LTEA) standard, transmit diversity is employed at the user terminal [3] to improve reliability.

The Alamouti code has linear ML decoding complexity at full spatial diversity order making it an attractive choice for SCFDMA since it is highly complex to perform full ML decoding. Using the conventional Alamouti space-time block code (STBC) for highly-mobile users where the channel changes rapidly from one SC-FDMA symbol to another, causes substantial degradation in performance. Earlier works on SFBC design in SC-FDMA include [4] and [1]. They focus on PAPR of the codes involved but do not address the issue of mobility. At high user mobility, the inter-carrier interference (ICI) due to Doppler results in significant performance degradation. While SC-FDMA is more robust to Doppler than OFDMA, for high user velocities of the order of $350 \mathrm{kmph}$ which must be supported by LTE, channel tracking and ICI compensation are needed for reliable decoding in SC-FDMA.

In this paper, we propose an effective SFBC scheme that mitigates ICI in high-mobility scenarios with a low decoding complexity. The scheme builds on our earlier work in [5] for OFDM systems

This work was supported by gift from RIM Inc. by investigating the design tradeoffs involved in mobile SC-FDMA with channel estimation effects. More specifically, varying the SFBC codewrod frequency span parameters results in interesting design tradeoffs between error rate performance, pilot overhead, and PAPR. Moreover, the proposed scheme allows for a distributed comb-type pilot structure unlike the LTE block-type pilot structure which is not effective in tracking the rapid channel variations under high mobility.

The paper is organized as follows. In Section II, we present the proposed SFBC SC-FDMA scheme. In Section III, we derive the transmitted SFBC-encoded signal model and evaluate its PAPR. We investigate the design tradeoffs in Section IV. Simulation results are presented in Section $\mathrm{V}$ followed by the concluding section.

Notation: $((.))_{N}$ indicates indexing with modulo $N$. Upper case boldface $\mathbf{A}$ represents a matrix whose $(n, m)$ element is $A(n, m)$ and lower case boldface $\mathbf{x}$ represents a vector whose $n$ th element is denoted by $x(n)$. $[\mathbf{A}, \mathbf{B}]$ represents concatenation of matrices/vectors column-wise and $[\mathbf{A} ; \mathbf{B}]$ represents concatenation of matrices/vectors row-wise. $\mathbf{F}_{N}$ denotes the Discrete Fourier Transform (DFT) matrix of size $N$. The constant $i=\sqrt{-1}$ and $(.)^{*},(.)^{H},(.)^{T}$ represent, respectively, the conjugate, Hermitian and transpose operators.

\section{SFBC DESIGN FOR MOBILE SC-FDMA}

The proposed SFBC scheme is shown in Fig. 2. It is similar to the scheme in [5] except that, with SC-FDMA, the modulation is performed in the time-domain and the SFBC encoding is done in the frequency-domain after the DFT. Assume that each user is allocated $M$ data sub-carriers, then $M$ symbols $\bar{s}(m) ; m=0, \cdots, M-1$ are generated in the time-domain and transformed to the frequencydomain resulting in $M$ complex symbols $s(m)$. We implement the Alamouti SFBC using the "super-block" structure shown in Fig. 1. The sequence for Antenna-1 is unperturbed and hence its PAPR is not affected. However, in Antenna-2, sub-blocks of $L$-symbols are swapped as shown and alternate sub-blocks are negated and the whole sequence is conjugated.

Each super-block is comprised of $2(L+D)$ sub-carriers where $2 L$ is the number of data sub-carriers in a super-block and $2 D$ is the number of pilot sub-carriers. The dark boxes in Fig. 1 represent the sub-carriers for pilot insertion which help not only in channel estimation but also avoid intra and inter-block interferences between Alamouti code blocks due to mobility-induced ICI. The value of $D$ is determined by the velocity of the mobile terminal where higher Doppler results in higher ICI. Super-block $q$ occupies sub-carriers $2 q(L+D)$ to $2(q+1)(L+D)-1$ with $q=0, \cdots, Q-1$ where $Q=\frac{M}{2 L}$. Therefore, the total number of sub-carriers allocated to a user including pilots is given by $K=M+2 Q D$. 


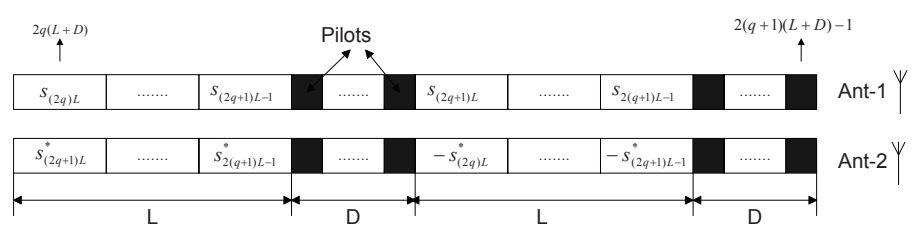

Fig. 1. Proposed super-block strucuture

Finally, we pad an appropriate number of zeros and take an $N$-point IDFT resulting in the time-domain sequence for each of the transmit antennas. After these operations, the sequence for Antenna- $d$ is denoted by $x_{d}(m)$ in the frequency-domain and $\bar{x}_{d}(n)$ in the timedomain for $m, n=0, \cdots, N-1$ and $d=1,2$. Moreover, for simplicity, we assume that a user's allocation starts with Sub-carrier 0 .

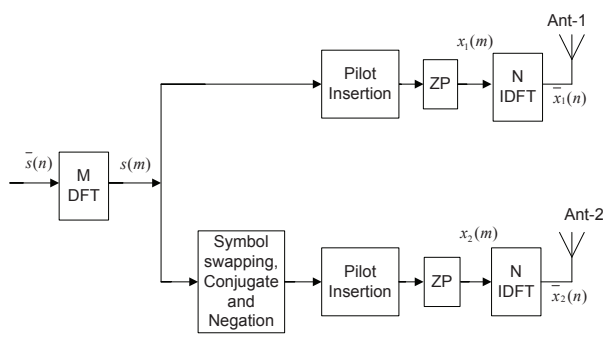

Fig. 2. Schematic of the proposed SFBC SC-FDMA scheme

\section{SIGNAL MODEL}

In this section, we derive the PAPR for the SFBC signal described in Section II. We do it separately for each antenna and use matrix notation for compactness. First, let us consider the symbol swapping and negation operation of Antenna-2. This can be represented using a matrix $\mathbf{W}_{(M \times M)}$ where the $(j, k)$ element of $\mathbf{W}$ is given by

$$
W(j, k)= \begin{cases}1, \quad & j-k=-L, j=2 q L+l, \\ & q=0, \cdots, Q-1, l=0, \cdots, L-1 \\ -1, \quad & j-k=L, j=2 q L+l, \\ & q=0, \cdots, Q-1, l=l, \cdots, 2 L-1 \\ 0, \quad \text { else } & \end{cases}
$$

As an example, for $M=12, L=3, Q=2$, we have

$$
\mathbf{W}=\left[\begin{array}{cc}
\mathbf{J} & \mathbf{O}_{6} \\
\mathbf{O}_{6} & \mathbf{J}
\end{array}\right] \text { where } \mathbf{J}=\left[\begin{array}{cc}
\mathbf{O}_{3} & \mathbf{I}_{3} \\
-\mathbf{I}_{3} & \mathbf{O}_{3}
\end{array}\right]
$$

The pilot insertion operation can be represented using a modified identity matrix where all-zero rows are inserted at positions corresponding to the pilot locations. Let us denote this matrix by $\mathbf{V}_{(K \times M)}$ whose $(j, k)$ element is given by

$$
V(j, k)= \begin{cases}1, & j-k=q D ; q=0, \cdots, 2 Q-1 \\ 0, & \text { else }\end{cases}
$$

Moreover, to include pilots, we define the matrix $\mathbf{G}_{(K \times 2 Q D)}$ whose $(j, k)$ element is given by

$$
G(j, k)= \begin{cases}1, & j-k=(q+1) L ; q=0, \cdots, 2 Q-1 \\ 0, & \text { else }\end{cases}
$$

Finally, we need to represent the ZP and upsampling operations which are essentially $\mathrm{ZP}$ in the frequency domain. Let the upsampling factor be $\gamma$. Then, we need to append $N^{\prime}-K$ zeros where $N^{\prime}=N \gamma$ and perform the IDFT. This ZP operation is denoted using the matrix

$$
\mathbf{Z}_{\left(N^{\prime} \times K\right)}=\left[\begin{array}{c}
\mathbf{I}_{K} \\
\mathbf{O}_{\left(N^{\prime}-K\right)}
\end{array}\right] .
$$

The time-domain equivalent signal $\overline{\mathbf{x}}_{1}$ for Antenna- 1 after pilot insertion and ZP is linearly related to the original signal $\overline{\mathbf{s}}$ as

$$
\begin{aligned}
\overline{\mathbf{x}}_{1} & =\mathbf{F}_{N^{\prime}} \mathbf{Z} \mathbf{V F _ { M }} \overline{\mathbf{s}}+\mathbf{F}_{N^{\prime}} \mathbf{Z} \mathbf{G s}_{t_{1}} \\
& =\left(\mathbf{U}_{1}\right)_{\left(N^{\prime} \times M\right)} \overline{\mathbf{s}}+\left(\mathbf{U}_{t}\right)_{\left(N^{\prime} \times 2 Q D\right)} \mathbf{s}_{t_{1}},
\end{aligned}
$$

where $\overline{\mathbf{s}}_{t_{1}}$ is the training sequence of length $2 Q D$. To evaluate $\mathbf{U}_{1}$ in (4), we observe that $\mathbf{F}_{N^{\prime}} \mathbf{Z V}$ is of size $\left(N^{\prime} \times M\right)$ and would contain those $M$ columns of $\mathbf{F}_{N^{\prime}}$ which have the indices $\alpha_{q}^{(0)}(l)=q(L+D)+l ; l=0, \cdots, L-1 ; q=0, \cdots, 2 Q-1$. These column indices of $\mathbf{F}_{N^{\prime}} \mathbf{Z V}$ would match with the row indices of $\mathbf{F}_{M}$ given by $\beta_{q}^{(0)}(l)=q L+l ; l=0, \cdots, L-1 ; q=0, \cdots, 2 Q-1$ and hence

$$
\begin{aligned}
U_{1}(n, m) & =\frac{1}{N^{\prime} M} \sum_{q=0}^{2 Q-1} \sum_{l=0}^{L-1} e^{i \frac{2 \pi n \alpha_{q}^{(0)}(l)}{N^{\prime}}} e^{-i \frac{2 \pi \beta_{q}^{(0)}(l) m}{M}} \\
& =\left\{\begin{array}{lc}
\sqrt{\frac{M}{N^{\prime}}} & m=n=0 \\
\frac{1}{\sqrt{N^{\prime} M}} & \frac{1-e^{i 2 \pi \theta L}}{1-e^{i 2 \pi \theta}} \frac{1-e^{i 4 \pi \theta Q L} e^{i \frac{4 \pi Q D n}{N}}}{1-e^{i 2 \pi \theta L} e^{i \frac{2 \pi D n}{N}}}, \quad \text { else }
\end{array}\right.
\end{aligned}
$$

where $\theta=\frac{n}{N^{\prime}}-\frac{m}{M}$. In a similar manner, we can see that

$U_{t}(n, q D+l)=e^{i \frac{2 \pi}{N^{\prime}} n \epsilon_{q}(l)}, q=0, \cdots, 2 Q-1 ; l=0, \cdots, L-1$

where $\epsilon_{q}(l)=(q+1) L+q D+l$ represent the pilot indices for super-block $q$. Therefore, we have

$\bar{x}_{1}(n)=\sum_{m=0}^{M-1} U_{1}(n, m) \bar{s}(m)+\sum_{q=0}^{2 Q-1} \sum_{l=0}^{D-1} e^{i \frac{2 \pi n \epsilon_{q}(l)}{N^{\prime}}} s_{t_{1}}(q D+l)$

Similarly, the time-domain equivalent signal $\overline{\mathbf{x}}_{2}$ for Antenna- 2 after symbol swapping, pilot insertion and ZP is linearly related to the original signal $\overline{\mathbf{s}}$. We first write $\overline{\mathbf{x}}_{2}$ as

$$
\overline{\mathbf{x}}_{2}=\mathbf{F}_{N^{\prime}}^{H} \mathbf{Z V W} \mathbf{s}^{*}+\mathbf{U}_{t} \mathbf{s}_{t_{2}}
$$

where $\mathbf{s}^{*}=\left(\mathbf{F}_{M} \overline{\mathbf{s}}\right)^{*}=\mathbf{F}_{M}\left(\mathbf{F}_{M}^{H} \mathbf{F}_{M}^{*}\right) \overline{\mathbf{s}}^{*}=\mathbf{F}_{M} \mathbf{R}_{(M \times M)} \overline{\mathbf{s}}^{*}$ where $\mathbf{R}$ is the reflection matrix given by

$$
R(j, k)= \begin{cases}1, & j=M-k \\ 0, & \text { else }\end{cases}
$$


and, therefore, we can write $\overline{\mathbf{x}}_{2}$ in terms of the conjugate of the original time-domain signal $\overline{\mathbf{s}}^{*}$ as

$$
\begin{aligned}
\overline{\mathbf{x}}_{2} & =\mathbf{F}_{N^{\prime}}^{H} \mathbf{Z V W} \mathbf{F}_{M} \mathbf{R} \overline{\mathbf{s}}^{*}+\mathbf{U}_{t} \mathbf{s}_{t_{2}} \\
& =\left(\mathbf{U}_{2}\right)_{\left(N^{\prime} \times M\right)} \mathbf{R} \overline{\mathbf{s}}+\mathbf{U}_{t} \mathbf{s}_{t_{2}} .
\end{aligned}
$$

where $\mathbf{s}_{t_{2}}$ is the training signal for Antenna-2. Using an approach similar to the one we used for Antenna-1, we see that the matrix $\mathbf{F}_{N^{\prime}}^{H} \mathbf{Z V W}$ contains those columns of $\mathbf{F}_{N^{\prime}}^{H}$ that have the indices $\alpha_{q}^{(1)}(l)=2 q(L+D)+l, \alpha_{q}^{(2)}(l)=(2 q+1)(L+D)+l ; q=$ $0, \cdots, Q-1 ; l=0, \cdots, L-1$. Corresponding to these columns are the rows of $\mathbf{F}_{M}$ whose indices are given $\beta_{q}^{(1)}(l)=(2 q+1) L+$ $l, \beta_{q}^{(2)}(l)=2 q L+l ; q=0, \cdots, Q-1 ; l=0, \cdots, L-1$ and hence

$$
\begin{aligned}
U_{2}(n, m) & =\frac{1}{N^{\prime}} \sum_{q=0}^{2 Q-1} \sum_{l=0}^{L-1}\left(e^{i \frac{2 \pi n \alpha_{q}^{(1)}(l)}{N^{\prime}}} e^{-i \frac{2 \pi \beta_{q}^{(1)}(l) m}{M}}\right. \\
& \left.-e^{i \frac{2 \pi n \alpha_{q}^{(2)}(l)}{N^{\prime}}} e^{-i \frac{2 \pi \beta_{q}^{(2)}(l) m}{M}}\right) \\
& =U_{1}(n, m)\left(e^{-i \frac{2 \pi m L}{M}}-e^{i \frac{2 \pi n(L+D)}{N}}\right) .
\end{aligned}
$$

Finally, we have

$$
\begin{aligned}
\bar{x}_{2}(n) & =\sum_{m=0}^{M-1} U_{2}(n, m) \bar{s}^{*}((M-m))_{M} \\
& +\sum_{q=0}^{2 Q-1} \sum_{l=0}^{D-1} e^{i \frac{2 \pi}{N^{\prime}} n \epsilon_{q}(l)} s_{t_{2}}(q D+l) .
\end{aligned}
$$

Now, the PAPR for each antenna is defined as

$$
P_{d}=\frac{\max _{n}\left|\bar{x}_{d}(n)\right|^{2}}{\frac{1}{N^{\prime}} \sum_{n=0}^{N^{\prime}-1}\left|\bar{x}_{d}(n)\right|^{2}}, \quad d=1,2
$$

The PAPR given by (14) is a random variable. In order to compute PAPR as a function of $L$, it is useful to evaluate the complimentary cumulative distribution function (CCDF) at a given probability for each $L$. If the CCDF is given by $C_{d}\left(P_{0}\right)=\operatorname{Pr}\left(P_{d}>P_{0}\right)=p_{T}$ where $p_{T}$ is a desired probability threshold and $P_{0}$ is the value of $P_{d}$ that achieves this threshold which we call the critical PAPR, then $C_{d}\left(P_{0}\right)$ can be used for comparison. Moreover, since we want to quantify the increase in PAPR due to the SFBC and not the actual PAPR itself, it makes sense to compare it against simple SCFDMA, If we denote the critical PAPR's for SFBC and SC-FDMA by $P_{0}^{(\mathrm{SFBC})}$ and $P_{0}^{(\mathrm{SCFDMA})}$ respectively, then the ratio $\delta=$ $P_{0}^{(\mathrm{SFBC})} / P_{0}^{(\mathrm{SCFDMA})}$ can be used as a parameter to characterize the SFBC scheme. In Section IV, we use $\delta$ to penalize the SNR since larger PAPR means either larger energy spent per bit or more errors. Since it is difficult to obtain the closed form PAPR-CCDF for SCFDMA systems, we resort to simulation results wherein a case study is presented in Section V.

\section{DESIGN TRADEOFFS}

In this Section, we describe the involved trade-offs between BER performance, pilot overhead and PAPR as the code design parameter $L$ is varied. In the super-block structure, we assume that the channel is constant over $L+D$ sub-carriers. But in reality, the channel is not constant over $L+D$ sub-carriers, affecting the orthogonality of the Alamouti code. Therefore, the performance degrades depending upon the correlation between the channel response at sub-carriers separated by $L+D$ which, in turn, depends on the delay spread of the channel. A similar problem has been analyzed for time-selectivity in [6], where the authors derive the distribution of the SINR $\mu$ as a function of correlation factor $\rho$ between the channels at two instants, which is given by $p_{\mu}(\mu)=\lambda^{-(\nu-\eta) \mu}(1+\eta \mu)$, where $\eta=2|\rho|^{2}\left(1-|\rho|^{2}\right) \frac{E_{s}}{N_{0}}$, $\lambda=\frac{2 N_{0}}{\left(1-|\rho|^{2}\right) E_{s}}, \nu=\frac{2 N_{0}}{\left(1-|\rho|^{2}\right) E_{s}}$ and $\rho$ is the correlation factor. Using $p_{\mu}(\mu)$, we can compute

$$
E\{\mu\}=\frac{E_{s}}{2 N_{0}}\left(1+|\rho|^{2}\right), \quad 0 \leq|\rho|<1 .
$$

For our case, $\rho$ is a function of the channel delay spread and the factor $(L+D)$. We denote the length of the channel impulse response (CIR) vector $\overline{\mathbf{h}}=\left[\bar{h}(0), \bar{h}(1), \cdots, \bar{h}(\tau-1), \mathbf{0}_{N-\tau}^{T}\right]^{T}$ by $\tau$. We assume that the CIR taps are circularly symmetric complex Gaussian random variables and are uncorrelated, i.e. $E\left\{\bar{h}(j) \bar{h}^{*}(k)\right\}=\sigma_{k}^{2} \delta(j-k)$ where $\sigma_{k}^{2}$ is the energy of tap $k$. We also assume $\sum_{k=0}^{\tau-1} \sigma_{k}^{2}=1$. Now, we need to calculate $\rho=$ $E\left\{h(j) h^{*}(k)\right\}$ where $\mathbf{h}=[h(0), h(1), \cdots, h(N-1)]^{T}=\mathbf{F}_{N} \overline{\mathbf{h}}$ and $k=j+(L+D)-1$. Using properties of complex Gaussian random vectors and the DFT, we obtain

$$
\rho=\sum_{k=0}^{\tau-1} \sigma_{k}^{2} e^{-i \frac{2 \pi k}{N}(L+D-1)} .
$$

For example, consider the channel with $\sigma_{k}^{2}=\frac{1}{\tau}, 0 \leq k<\tau$ and set the parameters $N=512, D=0$. When $\tau=1$, i.e., when the frequency response is flat, $|\rho|=1 \forall L$. For the case of $\tau=30, L=$ $10,|\rho|$ is as low as 0.6 and hence the SFBC should have a smaller $L$. Unfortunately, with interleaving and channel coding, a closedform expression cannot be derived for BER/FER performance as a function of $\rho$; therefore, we rely on simulations. Next, we penalize the SNR using the $\delta$ parameter defined in Section III as

$$
\operatorname{SNR}_{e}(L, D)=\frac{1}{\delta} \frac{E_{s}}{2 N_{0}} .
$$

Finally, we need to account for rate loss due to the insertion of pilots since the number of pilots changes with the parameters $L$ and $D$. Towards this end, we define one SC-FDMA symbol as one frame which has a frame error rate FER. Assuming $E_{s}=E_{p}$, the data rate in bits/second (bps) is given by

$$
\Gamma=\frac{M L}{N(L+D)}(1-\text { FER })\left(\log _{2} \xi\right) \psi B_{w}
$$

where $\xi$ is the signal constellation size, $\psi$ is the channel code rate, $B_{w}$ is the transmission bandwidth in $\mathrm{Hz}$.

\section{SIMULATION RESULTS AND DISCUSSION}

Similar to LTE, the carrier frequency and bandwidth were chosen to be $2.5 \mathrm{GHz}$ and $7.68 \mathrm{MHz}$, respectively. Other simulation parameters are $N=512, M=96$ (12 resource blocks). The multiple access mode was localized frequency division multiple access (LFDMA) and modulation scheme was 16-QAM. Our frame structure differs from that of the LTE standard mainly in using distributed comb-type pilots instead of block-type pilots. The channel estimation and tracking is based on the method proposed in [7] and hence we need snapshots of channel over the entire bandwidth. When there are multiple users sharing the uplink frame, the number of pilot sub-carriers need not be increased, but they are frequency or time multiplexed among the users. The mobile station is assumed 


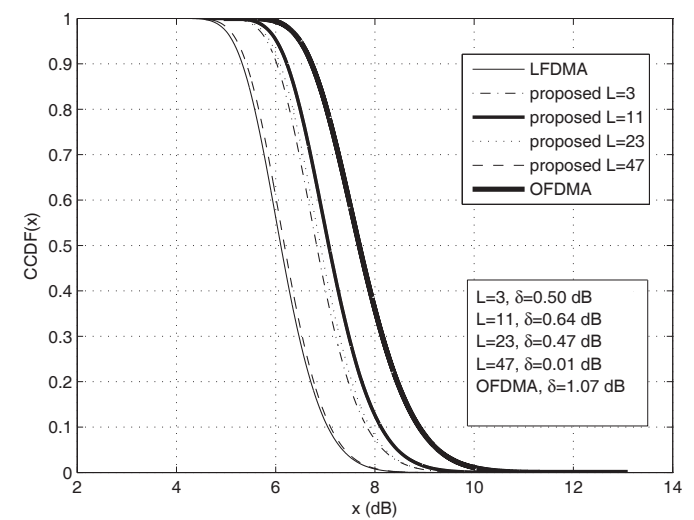

Fig. 3. PAPR CCDF for Different L

to be moving at a speed of $350 \mathrm{kmph}$. In order to be compliant with the RB sizes in LTE, we set $D=1$ and $L=3,11,23$ or 47 . The Stanford University Interim-3 (SUI-3) channel model was used and rate- $\frac{1}{2}$ convolutional coding with generator polynomial $[171,133]$ and random interleaving were employed. Shown in Fig. 3 are the CCDF's for the proposed SFBC scheme for different values of $L$. They have been compared against conventional SC-FDMA and OFDMA schemes. Also on this figure, we have listed values of $\delta$ for antenna- 2 for different $L$ at a probability of $p_{T}=10^{-4}$. The simulation was done with 1000,000 symbols. It can be seen that the SNR loss for our proposed SFBC SC-FDMA due to increased PAPR (compared to conventional SC-FDMA) is about $0.54 \mathrm{~dB}$ for $L=3$ and becomes negligible for $L=47$. Fig. 4 depicts frame error rate (FER) (including channel estimation effects) against SNR penalized with $\delta$ for different $L$ and assuming a 3-tap frequencydomain equalizer for each sub-carrier to compensate for ICI [5]. It can be seen that the best FER is achieved for $L=3$ where the SFBC codewords maintain their orthogonality and hence achieve higher diversity than higher values of $L$. Finally, in Fig. 5, we plot the rate $\Gamma$ in (18) for different $L$ where we see that $L=3$ and $L=11$ achieve the highest rate at low SNR and high SNR, respectively. This is because at low SNR where noise dominates ICI, the $L=3$ case has better FER than $L=11$ case though it has larger pilot overhead while at high SNR, the FER's for both cases improve and the low pilot overhead of $L=11$ case results in better rate. However, as $L$ is further increased to $L=23$, the channel can no longer be assumed constant within the SFBC codewords which degrades the FER and reduces the rate.

\section{CONCLUSIONS}

We presented an attractive SFBC scheme for the SC-FDMA uplink and demonstrated its robustness over doubly-selective channels with a controlled PAPR and low detection complexity. Using simulations, we studied the tradeoffs between training overhead, PAPR and loss of orthogonality due to the time and frequency selectivities of the channel. Simulation results demonstrated the viability of our proposed SFBC scheme for the SC-FDMA uplink in LTE-A. Using the same simulation procedures, the designer can determine the frequency spacing between the 2 information symbols in each Alamouti SFBC codeword. Our scheme can be directly extended to four transmit antennas using the rate $\frac{3}{4}$

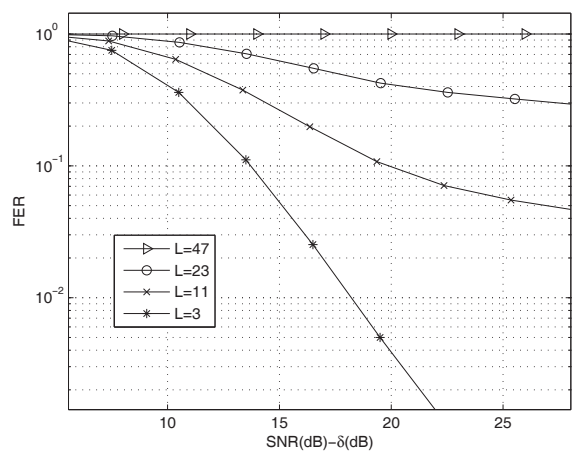

Fig. 4. FER vs. Effective SNR

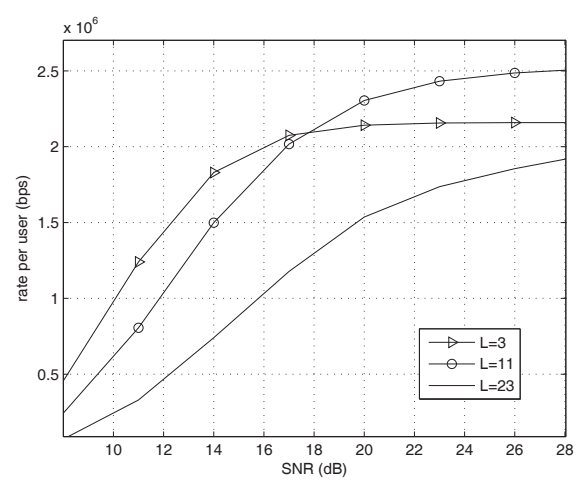

Fig. 5. Rate $\Gamma$ (per user) as a function of SNR for different $L$

Octonion orthogonal design at the expense of a small rate loss. To avoid the rate loss, the quasi-orthogonal code can be used at the expense of increased detection complexity. We are currently investigating the design tradeoffs for four transmit antennas.

\section{REFERENCES}

[1] C. Ciochina, D. Castelain, D. Mottier, and H. Sari, "A novel space-frequency coding scheme for single carrier modulations," in The 18th annual IEEE symposium on personal, indoor and mobile radio communications, 2007.

[2] Technical specification group radio access network, Physical channels and modulation, 3GPP TS 36.211 V8.1.0, Nov. 2007.

[3] Technical specification group radio access network, LTE-A trasmit diversity schemes for PUCCH format 1/1a/1b, R1092340, Jul. 2009.

[4] N. Tavangaran, A. Wilzeck, and T. Kaiser, "MIMO SCFDMA system performance for space time/freqeuncy coding and spatial multiplexing," in International ITG Workshop on smart antennas, Feb. 2008, pp. 382-286.

[5] S. Lu, B. Narasimhan, and N. Al-Dhahir, "A novel SFBCOFDM scheme for doubly selective channels," IEEE Trans. Veh. Tech., vol. 58, no. 5, pp. 2573-2578, June 2009.

[6] A. Vielmon, Y. Li, and J. R. Barry, "Performance of alamouti transmit diversity over time-varying rayleigh-fading channels," IEEE Journal Wireless Comm., vol. 3, no. 5, pp. 1369-1373, September 2004.

[7] Y. Mostofi and D. C. Cox, "ICI mitigation for pilot-aided OFDM mobile systems," vol. 4, pp. 765-774, Mar. 2005. 